\title{
Tipos y lugares de la enfermedad en la obra de Giuseppe Bonaviri
}

\author{
Vicente González Martín` \\ Universidad de Salamanca
}

\begin{abstract}
Resumen
La temática de la enfermedad en la historia de la literatura en general e italiana en particular tiene una larga historia, porque está radicada en la parte más profunda del ser humano desde el nacimiento hasta la muerte y condiciona, en muchos casos el recorrido vital de un escritor. Es el caso de Giuseppe Bonaviri, nacido en Mineo en 1924 y muerto en Frosinone en el 2009. Escritor siciliano amante de su tierra, de su familia y de sus amigos, fue una persona de frágil constitución física. Quizá por esta razón y por su profesión de médico, la enfermedad acompaña siempre al escritor siciliano en su vida y en su literatura y su lenguaje poético se nutrirá abundantemente de los materiales que la enfermedad y la medicina le ofrecen.
\end{abstract}

\section{Palabras clave}

· Enfermedad · Medicina · Hospital · Curación · Muerte · Fármacos - Sicilia · Familia

\section{Abstract}

The theme of illness in the history of literature in general and in Italian literature, in particular, goes back a long time because it is rooted in the deepest part of the human existence from birth to death, and in many cases it conditions the life-course of a writer, as it happened with Giuseppe Bonaviri, who was born in Mineo in 1924 and died in Frosinone in 2009. He was a Sicilian writer who loved his country, his family and his friends and a person of fragile physical constitution. Perhaps for this reason, and because of his profession as a medical doctor, illness always accompanied him in life and in literature, and his poetic language was abundantly nourished by the materials that both illness and medicine offered him.

\section{Keywords}

Illness · Medicine · Hospital · Cure · Death · Drugs · Sicily · Family

\footnotetext{
- Catedrático de Filología Italiana de la Universidad de Salamanca (España), ha sido Vicerrector, Director del Departamento de Filología Moderna, actualmente Decano de la Facultad de Filología, Presidente de la Sociedad Española de Italianistas, Director de la Cátedra Sicilia, Director de RSEI. Revista de la Sociedad Española de Italianistas, miembro de numerosas agencias españolas e internacionales de evaluación, ha publicado numerosos libros y artículos, de los cuales unos sesenta están dedicados a la literatura comparada italo-española.
} 
La temática de la enfermedad en la historia de la literatura en general e italiana en particular tiene ya un largo recorrido, porque la enfermedad se incardina en lo más profundo del ser humano desde que nace hasta su muerte y tińe, en muchos casos, el trayecto vital de un escritor, como veremos en el caso de Giuseppe Bonaviri, nacido en Mineo, en 1924 y muerto en Frosinone en el 2009. Escritor siciliano por los cuatro costados, amante de su tierra, de su familia y de sus amigos, fue una persona de frágil constitución física pero fuerte en sus convicciones humanas y literarias. Contribuyó en mucho al florecimiento de la literatura italiana escrita en Sicilia con un gran número de obras de diversos géneros, proyectándose fuera de su país con una obra consistente de alta literatura.

En el caso de Giuseppe Bonaviri, la enfermedad acompaña al insigne escritor siciliano en un amplio sentido. Abraza por completo su personalidad humana, pues él mismo se consideraba un ser enfermizo al que acechaban las enfermedades como algo consustancial a su naturaleza. Quienes hemos gozado de su amistad conocíamos bien esa preocupación que su siempre solícita mujer Lina intentaba alejar de él o, al menos, mitigar, generalmente sin éxito.

Quizá por esa tensión emocional frente a la enfermedad, rayana en la hipocondría, Bonaviri decidiera hacerse médico, estudiando de 1943 a 1949 la carrera de Medicina en la Universidad de Catania, ejerciendo primero de médico militar (subteniente médico) en Novara, después en Casale Monferrato y luego ejerciendo esta profesión en el hospital de Frosinone desde 1958 hasta su jubilación. La muerte de su padre por un ictus cerebral en 1964 a raíz del excesivo trabajo y la tensión emocional que esta profesión suscitó en él, le produjo un estado de ánimo ansioso-depresivo que lo acompañaría siempre.

El lenguaje poético elaborado por Bonaviri se caracteriza por un plurilingüismo, o polilingüismo, como algunos lo han definido, que colorea muchas de sus obras y en el que participan términos de muy diversas procedencias: desde el siciliano al español, al francés, ruso, inglés, y también tomados de diversos sectores de la actividad humana.

De ahí que el estudio y ejercicio profesional de la medicina implique en Bonaviri no solo un conocimiento profundo de la materia, sino también del lenguaje que emplea, y que nuestro autor no dudará en aprovechar intensamente para la elaboración de sus obras, tiñendo su lenguaje poético de términos médicos, casi siempre precisos y de gran eficacia literaria. Él mismo así lo declara, en una entrevista que Roberta Tiberia le hace en febrero de 2005:

Ho fatto il medico per sei anni in Sicilia; e per sei anni nel vecchio e poi nel nuovo ospedale di Frosinone. Nel vecchio, ero in infermeria: si facevano tre giorni alla settimana di trenta ore di guardia; ho imparato li a fare il raschiamento e l'assistenza ai ferri la notte. Questa esperienza mi ha fatto scendere nelle caverne del dolore umano, e mi ha permesso di venire a contatto con la conoscenza profonda del dolore vero e proprio, e non di quello che sta in superficie e che rimbalza subito nei problemi politici, sociologici, anche importanti; il vero dolore dell'uomo è quello biologico, della malattia, della morte. La mia esperienza ha allargato la mia visione e la mia possibilità di avere contatti più diretti e di penetrare meglio nelle pieghe più segrete dell'animo umano. Quindi in questo senso esiste un rapporto fra la pagina scritta 
e la medicina. Entrambe comportano un'immersione nel mondo del dolore umano, filtrato e disperso in tutti i miei libri. $(2005)^{1}$

Bonaviri, como tantos otros médicos escritores en todas partes del mundo -es curioso señalar que en Italia existe la Associazione Medici Scrittori Italiani (AMSI) están obligados en el ejercicio de su profesión a tratar a un gran número de personas enfermas, de diversa condición, edad y estado, y todos comparten el padecer una determinada enfermedad y el temor a la muerte. El médico ineludiblemente debe centrarse en el hombre, en su cuerpo y en su psiquis y lo hace en momentos de enorme tensión física y psíquica, en los que el paciente se siente muchas veces inerme y está dispuesto a dejar su cuerpo y su mente en manos del médico. De aquí la posibilidad que el médico tiene de conocer y diagnosticar los problemas del ser humano y ese diagnóstico puede dar lugar a la receta de un medicamento o una cura o un texto literario donde se plasme y se describa el dolor humano, tema decisivo en la creación literaria de todos los tiempos.

Con estos firmes avales, entre ellos el del propio Bonaviri, centraré principalmente en esta última vertiente el análisis en este estudio, que podría bien estructurarse temáticamente como sigue:

1) La enfermedad en general, cómo aparece en las obras, tipos y variedades de enfermedades, diagnósticos y curas, medicamentos, técnicas, etc.; en qué personajes aparece, cómo reaccionan ante ella, períodos en los que la presencia de la enfermedad es más o menos intensa.

2) Lugares en los que se desarrollan y curan las enfermedades.

3) Obras en las que las enfermedades y la presencia de la medicina son fundamentales.

4) Lenguaje médico que se incorpora a sus obras.

Cada uno de estos aspectos, estudiados exhaustivamente en las obras de Bonaviri, agotarían por sí solos el espacio disponible para el artículo. Así que ahora solamente analizaré algunos de ellos, los que considero más significativos.

\section{Enfermedades}

Son muchas las enfermedades reseñadas en las obras bonavirianas y no podemos ni siquiera mencionarlas ahora. Muchas son roturas de huesos o contracciones musculares producidas en el trabajo o por caídas en el caso de los viejos; cardiopatías, enfermedades de los ojos, flebitis, cáncer de estómago, trauma craneal, fiebre, fiebre de malta, fibroma, tos convulsa, trombosis cerebral, asma cardiaca, pleuritis, ictus, hemorragia de la retina, conjuntivitis, morbo contagioso, melancolía, depresión, esquizofrenia, hipocondría y, para terminar, la que sufre un personaje en el relato «Il Vecchio» de I cavalli lunari: «encefalopatía multinfartuale arteriosclerótica» (Bonaviri, 2004:39).

El proceso del parto, aunque no sea una enfermedad se presenta en diversas ocasiones como el prototipo del dolor profundo: «fiero dolore», que debe sufrir Pina y las demás mujeres parturientas de sus novelas. Y es un dolor que debe sufrirse, porque ni 
siquiera Jesús puede quitarlo y tampoco mitigarlo, compartido por todo el universo. Así nos dirá en Gesù a Frosinone, al abrir el abdomen de Pina en la sala quirúrgica: «Il sangue che ne venne fuori era il sangue di tutto il mondo» ${ }^{2}$ (2004:197).

La omnipresencia de la enfermedad hace que incluso Bonaviri construya poesías con sus propias enfermedades. Un buen ejemplo de ello es el poema L'emorragia retinica della luna, donde nuestro autor recrea poéticamente la hemorragia de retina que tuvo el 9 de agosto de 1994 en el ojo derecho por trombosis parcial de la vena retiniana central, traspasando su propia enfermedad a la luna, que, impedida por la enfermedad, pierde su fuerza gravitacional:

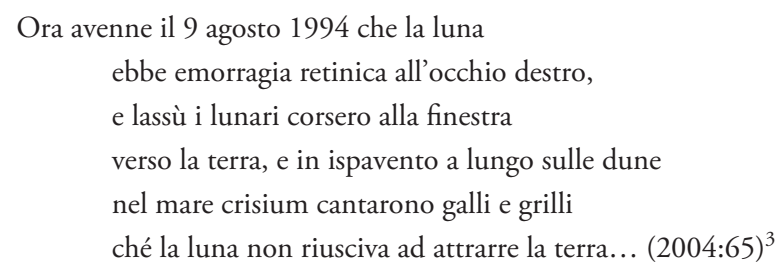

Las enfermedades que se reseñan a lo largo de las obras de Bonaviri son, generalmente, las somáticas, las que conciernen a las diversas partes del cuerpo humano, como hemos ido señalando. Sin embargo, en algunas obras comparecen enfermedades mentales o disquisiciones concernientes al funcionamiento del cerebro, que, si bien no son en sí mismas enfermedades, comparten en su descripción un lenguaje igual o semejante.

Esta temática, por ejemplo, es la central en la novela Il dormiveglia, escrita entre 1986 y 1987, en la que los personajes, en su mayoría científicos, incluido el propio Bonaviri, presente, como casi siempre como voz narrante, discuten e intentan dilucidar el misterio de ese estado que es el duermevela, surgido antes del sueño y de un pensamiento no racional. Previamente analizan enfermedades psíquicas como la neurosis ansiosa, cuyos síntomas son, según los personajes, un malestar que se convierte en inquietud, la idea de que una fuerza psíquica externa se quiere introducir dentro, angustia, etc.

La obra termina con un Apéndice titulado "Osservazioni teoriche sul dormiveglia (scritte da Epaminonda, mentre, andando, per monti, acque abissi e lune, seguiva il filo del suo pensiero canterino)» (1988:233).

En este breve opúsculo Epaminonda reflexiona sobre los dos tipos de pensamientos presentes en el hombre: el racional y el nocturno y de la obsesión como base de fondo. Epaminonda, personaje de ficción a todos los efectos, utiliza un lenguaje científico verosímil para el argumento que defiende, pero en el que se integran también elementos ficticios bajo apariencia de cientificidad. En definitiva, es lo que Bonaviri produce con este tipo de lenguaje en todas las situaciones en que lo utiliza. Veamos cómo define el pensamiento nocturno, o de duermevela:

Questo secondo pensiero si manifesta e si attivizza, nella fase dormivegliante, o pre-onirica. Al di fuori è inconcepibile e inesistente. La scaturigine di fondo di questo pensiero notturno è, in gran parte, sensoriale. È sufficiente autocontrollarsi, prima di addormentarsi, per capirlo. Basta che, durante il pre-sonno, sottostiamo a stimoli cutanei, o viscerali in senso lato (prurigini, somatostesie, algie, semplici psicostesie) perché, in un gioco de afferenze corticali e di efferenze encefalo — cutanee, o viscerali, si presenti questo pensiero ${ }^{4}$. (108) 
Epaminonda concluirá aceptando que el pensamiento duermevelante se produce y posee una obsesión de fondo que se somatiza en enfermedad y que se concentra esencialmente y se visualiza en la piel:

In questo abbozzo interpretativo del dormiveglia mi rifaccio all'ipotesi somatico-viscerale, escludendovi beninteso i dinamismi del sogno. Considero, per esempio, il mantello cutaneo come una espansione all'in fuori della stessa massa encefalica. Il rivestimento cutaneo, vero guscio pericorporeo che ci ingloba, si può, quindi, ritenere un'appendice, espansa all'in fuori, del cervello, cioè un vero mantello mini-encefalico che possiede una proto funzione arcaica micro-pensante. D'altronde, non si dimentichi che la membrana cellulare ha una reattività nervosa primordiale. La cute potrebbe anche possedere una primigenia funzione memorizzatrice ${ }^{5}$. (212)

El perfecto acople entre narración y aprovechamiento de las enfermedades físicas y mentales se produce, tanto en lo que se refiere a los autores como al texto, en la obra que Giuseppe Bonaviri escribe junto a su hija Giuseppina Bonaviri, titulada E il verde ramo oscillo. Fiabe di folli.

Dos médicos, uno cardiólogo y la otra psiquiatra, se unen para convertir en literatura dos esferas constituyentes del ser humano que se confunden en un sanatorio donde los médicos intentan con los enfermos «autocurarli attraverso le fiabe che loro stessi, a ritmi distanziati, e su nostro invito, ci hanno narrato. Ossia potremmo parlare di una terapia fiabologica, o fiabaterapia» (Bonaviri, 1999:7). Allí psicólogos y psiquiatras intentan analizar y curar con las técnicas tradicionales a los esquizofrénicos hospitalizados y se dan cuenta de que poco o nada saben sobre los complejos problemas de la mente humana y que las soluciones biológicas por sí sola no sirven para curar las mentes enfermas.

Por lo que se refiere a los medicamentos, la problemática es muy variada pues el médico sabe qué medicina tiene que recetar para las diversas enfermedades pero choca muchas veces con la negativa del paciente, que siente aversión por lo que le han recetado por causas diversas y contra la administración, que se resiste a prescribir determinados medicamentos porque son caros. Reproduzco uno de los diálogos más significativos en este sentido del médico con el alcalde:

Per le milletrecento persone iscritte nell'elenco dei poveri, il medico condotto, che sostituivo frequentemente, poteva soltanto ricettare vecchi preparati galenici che rimontavano a cent'anni addietro

- Potete curare oggi un bambino affetto da bronco-polmonite con l'infuso di poligala?

— Gli infusi sono sempre buoni.

— E gli antibiotici non esistono? Se fosse un vostro fratello, cosa fareste?

— Dottore, se ci facciamo prendere la mano dalla pietà cristiana, è finita. Il comune va a rotoli. Non sapete quanti milioni di deficit abbiamo?

...Fate almeno che si possa prescrivere liberamente la penicillina. $(2010: 132-133)^{6}$

\section{Lugares de la enfermedad}

La comparecencia física de la enfermedad en las obras de Bonaviri suele producirse fundamentalmente en dos espacios: los hospitales y las casas, ambos receptáculos de los enfermos y 
representativos de formas distintas de entender la enfermedad. Por lo general, el ambiente y el tiempo en el que viven la mayoría de los personajes bonavirianos es de pobreza, cuando no de miseria, material y cultural y la lucha contra la enfermedad encuentra resistencia por parte de muchos enfermos, que se resisten a abandonar sus casas y consideran el hospital como signo de gravedad y quizá de no retorno, como veremos.

Los enfermos que pululan en las obras de Bonaviri tienen casi siempre la pretensión de curarse en sus propias casas, porque suelen considerar que una parte importante de su salud proviene de los efluvios benignos que la naturaleza les ha proporcionado siempre. Sus campos, sus casas, aunque sean pobres e incluso miserables, sus animales, son los que les dan seguridad, los que preservan su salud, alimentan sus cuerpos y, por tanto, creen, sus almas.

Por otra parte, cuando los personajes viven en ambientes campesinos, con arraigadas costumbres y supersticiones, desconfían del médico, de las curas y, sobre todo, de los medicamentos que este les prescribe. Bien porque prefieren la medicina tradicional, basada en tradiciones ancestrales y en la superstición con ribetes de magia, o bien porque no tienen dinero para pagar al médico y las medicinas y no esperan nada del estado, excepto el que le pidan más impuestos.

Para ver cómo se plasma esta situación en la literatura bonaviriana, señalaremos algunos pocos ejemplos.

En Silvinia (2007) se describe el proceso de enfermedad de la protagonista que da nombre a la novela. Se presenta acostada en el lecho de su casa, visitada por sus amigas y vecinas, en un ambiente semimágico, en el que los remedios médicos ancestrales sustituyen a los de la medicina clásica, por otra parte, casi inexistente en Idrisia.

Lo real y lo imaginario se confunden en un continuum de alucinaciones producidas por la morbosidad del ambiente y por las antiguas canciones de cuna que su madre, María Palermo, le canta continuamente porque cree que su curación depende de que duerma lo máximo posible: «Duerme, duerme, alma mía de mi corazón, sigue el camino que para ti ha trazado el Señor». (2007:116)

Muchos ejemplos de este tipo se encuentran en la novela L'enorme tempo (2010) escenificada en Mineo, prototipo de esa percepción de la enfermedad como algo ineludible, que el destino trae y que es difícil de eludir.

Los diversos casos que se relatan tienen en común que la llamada al médico suele ser de urgencia porque la enfermedad está ya muy avanzada y se itera la miseria del espacio de las casas y de las habitaciones donde se encuentran los enfermos, el sufrimiento estoico de la enfermedad y el rechazo a permanecer en la cama o ir al hospital, como hemos señalado, debido a que necesitan trabajar para comer. Al mismo tiempo que se manifiesta reticencia a comprar medicamentos, se destacan los esfuerzos del médico por curar a estos enfermos, a pesar de los pocos medios de los que dispone, así como el estoicismo de los familiares y vecinos cuando se produce un resultado fatal y el orgullo de los padres y familiares sin medios, que, sin embargo, juran pagar al médico como sea.

A modo de ejemplo, presentaremos dos casos referido uno a un niño, y, otro, a un viejo.

La historia del niño está datada en diciembre de 1949. El aviso urgente se lo da al médico un jorobado pariente del enfermo. Tras una carrera por las sinuosas 
calles de Mineo, esquivando las preguntas de los que se va encontrando, llega a la casa del paciente y comienza la descripción física:

L'ammalato era figlio d'un broccaio che abitava in un vicolo pieno di sterco e di pozzanghere, minime o larghe, ai bordi delle case. Salii, preceduto dal gobbetto, per una corrosa scaletta di legno, e lassù trovai il padre ad aspettarmi con in mano un lume a petrolio. Tenendo questo con ambedue le mani, enormi di calli, mi accompagnò per due stanze dai muri nudi preso cui c'erano sistemati molti letti...Il ragazzo stava seduto sul letto, appoggiato a due cuscini alti, ripieni di paglia. $(2010: 22)^{7}$

Rodeado de parientes y vecinos el médico diagnostica una cardiopatía y pacientemente oye a la madre contar cómo había inentado curarlo con «uno 'stomacale'di erba del vento e aceto da mettere sullo stomaco».

Revelador es el caso del canónigo, nominado N., tío de Peppi T., quien lo va a buscar para que urgentemente vea a su tío. El canónigo vive en una casa cerrada, en la que «El aire encerrado hedía»; vomita continuamente y su aspecto preanuncia la muerte: «L'aria trágica che si respirava là dentro è quasi impossibile di descrivere. Il canonico N. era magro, con le guance incavate e gli zigomi sporgenti: gli occhi erano acquosi e smorti, come quelli d'un animale colpito a morte» ${ }^{8}$ (2010:34).

Mientras el médico lo explora, el canónigo le da explicaciones sobre su situación. El diagnóstico es un cáncer de estómago que había estenotizado el píloro y, como es lógico, le recomienda que vaya a un hospital, pero el cura se aferra a su casa: «Sa, dottore, ho le mie abitudini e la mia casa è la mia casa. Lo sa bene lei, no?»" (2010:36). Finalmente, pasado un tiempo, acepta ir a un hospital y quince días después del ingreso morirá.

Los hospitales, descritos en su aspecto físico y humano con precisión, muchas veces en su estructura física y con empatía en lo que se refiere a los componentes humanos, se caracterizan generalmente por estructuras envejecidas, falta de medios humanos, almacenamiento de enfermos y, a pesar de todo, estimados por la ímproba labor que desarrollan sus trabajadores: médicos y resto de personal sanitario.

Los hospitales citados y descritos son el Fatebenefratelli, de Milán, los de Catania, Frosinone, un hospital y un asilo innominados de Nueva York y el hospital de pobres de Benarés, en la India.

En La ragazza di Casalmonferrato (2009) el hospital es el llamado Fatebenefratelli y se encuentra en Milán. Pino y Carla, personajes protagonistas van a él, concretamente a la planta de medicina interna, para visitar a Gianfranco, hermano de Pina que se encuentra hospitalizado. Siguiendo el iter acostumbrado en la descripción de estos lugares, el narrador nos sitúa espacialmente: «La sala era piccola con otto letti e da una finestra, dai vetri color latte, veniva un rumore lontano, indefinibile» (2009:170).

El paso siguiente es la presentación del componente humano del lugar, poniendo el foco en los demás enfermos y, sobre todo, en el médico, actor destacado y protagonista en el proceso de la enfermedad. Como en otras obras, se demuestra que en los hospitales se suele tratar a los pacientes y familiares con un cierto desdén, empleando con ellos un lenguaje frío, pero muy preciso y concreto:

Il dottore con uno sguardo distante: Ah, sì. È entrato da pochi giorni. Ha una pleurite essudativa. In appresso lo svuoteremo. Spero che il polmone sia indenne. Se è così, tutto guarirà in un 
mese. In caso contrario potrebbe trattarsi d'una tubercolosi che potrebbe durare a lungo. Era molto sciupato il ragazzo quando è entrato ${ }^{10}$. (2009:171)

Esa imperturbabilidad contrasta de forma muy visiva, casi siempre, con la angustia y la gestualidad de los pacientes y familiares.

En la misma novela, la protagonista Carla tiene que ser también ingresada en un hospital innominado.

En la novela L'enorme tempo (2010) en la que nos detendremos más adelante, se menciona, sin describirlo, el hospital de Catania. En esta ocasión, lo que importa es el ambiente interior, presentado por uno de los pacientes: Massaro Orazio, enfermo con «le caviglie e l'addome gonfio per scompenso di cuore» y a quien pesa tanto el salir para ayudar en el trabajo a su familia, como la angustia que le produce la soledad en la que se encuentra en un mundo para él extrańo y desconocido:

—Come mai avete lasciato l'ospedale? —gli chiesi dopo averlo visitato.

-Oh, dottore, voscenza sa che significa star chiuso notte e giorno in un palazzone dai grandi corridoi scuri? Nessuno vi sorride, nessuno vi guarda in faccia con compatimento, ed uno smania vedendo la grand'aria che c'è fuori e pensando alle nostre campagne ${ }^{11}$. (2010:28)

Los hospitales de Frosinone comparecen en diversas obras. Así, en el hospital geriátrico de Frosinone se desarrolla la acción de la obra Il giovin medico e don Chischiotte, de la que hablaremos después. A otro hospital de la misma localidad llega Jesús con sus discípulos en "Gesù a Frosinone» (2008:193). Y, en Il dormiveglia, el personaje-narrador, que se encuentra en el lejano oriente, recuerda desde la lejanía espacial y temporal su trabajo como asistente medico en el hospital de Frosinone, el que, en los primeros años de 1960, era, nos dirá, «un vecchio caseggiato fuligginoso chiuso da un muro dove a malapena cresceva la parietaria», para pacientes muy enfermos y terminales, viejos y niños, mezclados con todo tipo de enfermos y en pésimas condiciones ambientales y de salubridad:

Nelle corsie c'erano cardiopatici, bronchitici, tifosi, o persone affette da emopatie o da epatiti virali. Comunemente, gli scoli ematici, gli spurghi, non potendosi sempre smaltire attraverso le usuali condutture, erano incanalati nel vicino fiume Cosa, che ha un regime torrentizio, ed ha le sponde piene di gomme inservibili, di sacchetti di plastica, di gatti morti, di topi ${ }^{12}$. (1988:69)

Una pequeña mención dedica en la novela Silvina, para describir la estancia de Salvatore Casaccio (abuelo en la realidad de nuestro escritor) en un hospital de Nueva York, limitándose a situarlo con un breve apunte descriptivo: «Un vecchio casseggiato circondato da pini, adibito a ricovero di vecchi» (2007:165).

Con más precisión, en cambio, se describe el geriátrico en el que acaba el último tramo de su vida el viejo Casaccio, situado en una calle secundaria de la ciudad de Nueva York, desde la que se divisa Central Park — siempre los datos exactos de localización:

L'ospizio era una vecchia costruzione forse di fine Ottocento, adibita in passato a deposito di legna. Sul davanti aveva un giardinetto che contro i muri finiva a tre punte. Tuttora vi fiorivano stentatamente dei cespugli di rose sotto le quali se vedevano delle lucertole morte piene di petali ${ }^{13}$. (Ibíd.) 
En el elenco de los hospitales bonavirianos ocupa un puesto importante en la estructura de la novela $\dot{E}$ un rosseggiar di peschi e d'albicocchi (1986) el hospital de pobres de Benarés, en la India, calificado por el narrador de «nosocomio». Presenta un vistoso contraste entre la armonía de la construcción y la fealdad del contenido. $\mathrm{El}$ aspecto externo suscita sentimientos de equilibrio y belleza:

L'ospedale dei poveri, a Benares, si trova su una collina sotto la quale il tempio di Nakhan ha molte porte a foggia di rosa dei venti. Nei lunghi colonnati, fatti di pietra, si vede la dea Parvati che in pose diverse si unisci con i tori sacri... L'ospedale è costituito di tre padiglioni: quello per le donne, uno per gli uomini, e, infine, quello per i vecchi che arrivano con i volti affilati, e un involto di panini di riso ${ }^{14}$. (1986:29-30)

En el interior, jóvenes médicos inexpertos se sirven de manuales para intentar un diagnóstico correcto y la inmundicia y la estrechez de los espacios producen sensaciones de angustia:

Per ogni corsi c'è un solo bagno, i vasi con le urine vi vengono raccolti l'uno sull'altro per mancanza di spazio. In alcuni secchi si accumulano le placente delle partorienti da cui le mosche succhiano sangue. In un vecchio dipinto murale si legge: «Se la luna ti alluna la testa, prega Shiva. La nostra anima subisce corruzione eterna» ${ }^{15}$. (30)

La obra titulada Il giovin medico e Don Chisciotte (Comediola in due atti senza epilogo). Inserta en el Infinito lunare, es un prototipo de representación de la enfermedad en estado puro y de la incapacidad, en muchas ocasiones, de reaccionar a tiempo.

La acción de esta «comediola» se desarrolla en un viejo hospital «cronicario per vecchi», situado en Frosinone, que, además de los espacios destartalados, en su mayor parte, dedicados a los viejos, cuenta con «Neri sotterranei» donde «vengono raccolti i corpicini dei feti abortiti». Todo es tétrico. Incluso el sonar de las campanas es «un suono malinconico» y la luna no brilla igual que en otros lugares.

En este trágico, enfermizo y deprimente ambiente, desarrolla su labor de médico el joven Michele Rizzo. Pese a sus convicciones antiabortistas, de forma sorprendente, va a realizarse como persona y como médico, desde el momento en que recalan en él, cansados y fatigados por su largo peregrinar, Don Quijote y Sancho Panza.

A través del diálogo que se entabla entre el joven médico y los dos ilustres personajes se van a ir señalando y juzgando, a veces, los diversos componentes de la trama que confluyen, esencialmente, en las temáticas de la enfermedad, el dolor que esta conlleva y la muerte como consecuencia ineludible. Y todo ello se presenta con un lenguaje en el que el léxico, especialmente los epítetos, sirven para denotar lo tétrico del lugar.

Ya desde el comienzo Don Quijote se sorprende por el aspecto del «negro caseggiato" y por encontrar en él a una persona tan joven, al que inmediatamente pregunta:

IL MEDICO (dopo un po' risponde) Mi chiamo Michele Rizzo. El caseggiato è un cronicario. Per recenti disposizioni inoltre vi vengono raccolti i bambini mai nati.

DON CHISCIOTTE (che ha uno scudo color zafferano e una farfalla, per cravatta, color gelsomino). Il tuo trabajo... lavoro mi dà una grande tristezza. In questo luogo c'è solo morte?... è mortura infinita. (Piega il capo, affondando la lancia, dalla punta rossa, nel terreno) 
La primera reacción de Don Quijote es escapar de ese maléfico lugar, pero en su mente, siempre llena de fantasía, un pensamiento esquizofrénico lo lleva a identificar a Michele Rizzo con Miguel de Cervantes «incarcerato in quel ospedale».

A lo largo del diálogo, Don Quijote y Sancho se convierten en representantes de una civilización más libre en la que la enfermedad y el dolor constituyen algo natural y se enfrentan así a lo que representa el joven médico Michele Rizzo, como este mismo reconoce, en el hospital:

IL MEDICO (ancor più preso da quell'apparizione, dopo un pensoso silenzio dice) Sono un ragionier della morte. Solo a qualche vecchio riesco a ridar la vita. Gli altri, mentre muoiono, mandano riverberi sanguigni per queste finestre. $\mathrm{O}$ mio grande Don Chisciotte, venendomi a trovare tu mi ricordi la mia infanzia, fatta di vento, di miseria, di fiabe, di cieli stellati ${ }^{17}$. (2008:100)

La continua identificación que Don Quijote hace de Miguel de Cervantes con Michele Rizzo, lo lleva poco a poco a ir asumiendo el papel que el caballero andante le adjudica. Frente a la aparente locura del discurso, interviene, como garante de la cordura y la razón, el enfermero Raniero Marcoccia, aunque sin éxito.

La enfermedad predominante en este hospital no será la decrepitud de la vejez o las consecuencias de los abortos, sino la esquizofrenia contagiosa de Don Quijote y la confusión de las mentes entre la realidad y la ficción. Bonaviri se muestra aquí buen conocedor de Don Quijote y buen discípulo de su paisano Luigi Pirandello.

\section{Obras representativas de la enfermedad}

Algunas de las obras de Bonaviri tienen como temática principal la enfermedad y todo lo que la rodea. No es el momento de analizarlas con detenimiento ahora, pero al menos quiero presentarlas. La más importante, en cuanto a esta temática se refiere, es L'enorme tempo, escrita entre 1955 y 1961 y que, en realidad, es una especie de diario novelado. El protagonista es un joven médico narrante, alter ego de Bonaviri, quien desde el microcosmos de un pequeño pueblo, Mineo, contempla el fatigoso vivir de unas personas abocadas al duro trabajo y al dolor que las enfermedades le traen, casi sin posibilidad de curación. Porque la miseria, la forma de vida arcaica, la falta de cultura, obstaculizan continuamente los esfuerzos del médico, a pesar de que él es fruto de ese ambiente $y$, en principio, comparte la forma de ser y las costumbres de sus habitantes.

El propio Bonaviri describe la situación de partida de la obra en una entrevista para «Il Resto del Carlino», 14 diciembre 1976:

...quella fatta a Mineo, quando giovane venticinquenne vi arrivai come medico, ha una sua dimensione particolare. Mi calavo nel mio mondo nuovamente ma per altri canali di circolazione del dolore e della esperienza, raggiungevo insomma un tempo enorme dentro cui fermentavano esperienze millenarie di tragico sapore, di medicina alchemico-magica, di storie di pazienti l'uno all'altro legati, per funicoli ombelicali...Vi tornavo come medico, pronto a cogliere per coordinate razionali i segreti recessi del dolore contadino ${ }^{18}$. (Nigro en Bonaviri, 2010:12) 
En este lugar olvidado de la Sicilia comienza a ver lisiados por las calles; muchos viejos enfermos, viejas costumbres sanadoras con componentes mágicas, que no curan sino entorpecen la curación e incluso matan. Debe contrarrestar estos hábitos y para ello, el joven médico no contradice los argumentos del paciente por disparatados que sean, sino que distrae su atención y los tranquiliza con los paisajes y los sonidos que llegan del pueblo o del campo. También debe enfrentarse con la realidad de la enfermedad, dándose cuenta inmediatamente de lo difícil que es transmitir lo aprendido en la Facultad de Medicina a enfrentarse solo con los enfermos: «Quale abisso fra i corsi universitari e la pratica minuta e grigia e incerta d'ogni giorno" (2010:43).

La presencia de las fiebres de Malta y su firme decisión de atajarlas, a través de la vacunación y de la higiene, le lleva a enfrentarse con las autoridades primero y luego con los campesinos que no quieren vacunar a sus hijos ni a sus cabras y mucho menos dejar de beber la leche de las cabras.

También la enfermedad se convierte en el núcleo central de la comedia Il Giovin medico e Don Chisciotte, del que ya hemos hablado y del relato titulado Martedina, incluido en L'Infinito lunare, en I cavalli lunari y parcialmente en $\mathrm{Il}$ dottor Bilob.

$\mathrm{Y}$ en Martedina, una parte importante se centra en curar la enfermedad: los médicos, especialmente el protagonista, las enfermeras/os, los diversos tipos de enfermedades, las técnicas médicas que se emplean, la praxis y la ética médica, etc.

\section{Conclusiones}

Del análisis de las obras de las obras se deduce una serie de conclusiones que avalan el enorme peso que tiene la enfermedad y todo lo relacionado con ella tanto en la personalidad como en la escritura de Giuseppe Bonaviri.

Es muy significativo el hecho de que la voz narrante sea muy a menudo un trasunto del propio autor, que nunca se esconde lo suficiente detrás del personaje como para pasar desapercibido. Aún más, hay un interés consciente en manifestarlo, como si el escritor siciliano quisiera así afianzar la veracidad de los hechos y la omnipresencia en ellos de la familia y de la Sicilia que abandonó.

Los personajes protagonistas sienten una angustia vital que les agobia y que se genera por el desarraigo de la tierra materna y por el oficio de médico. Y ni siquiera son capaces de calmarlos los personajes bondadosos y prácticos, generalmente mujeres que los acompañan, como Martedina o Carla. El mal se les revela continuamente en la enfermedad y en el miedo que genera en la sociedad, que no la asume e intenta esconderla como si fuera algo vergonzoso o maligno en hospitales, asilos u otras instituciones. La máxima que se repite es que ciertos lugares es mejor que no se vean y que los enfermos deben estar lejanos de los sanos.

Probablemente esta actitud renuente hacia la enfermedad se explique también por la costumbre ancestral de muchos pueblos mediterráneos de no comunicar a los demás sus enfermedades, porque ello supone una especie de tacha para la familia; no un castigo, sino una pena que la gente pobre debe pagar.

Siguiendo esa línea de pensamiento, entre el médico y el enfermo se establece una relación de desconfianza, porque aquél no confía en la medicina oficial ni en los lugares donde se practica. Por ello no es raro que la mayoría de los enfermos 
pertenecientes a la clase trabajadora, sobre todo campesinos, piensen que el hospital no es un lugar a donde se va para curarse, sino para morir.

Especialmente trágica es la relación que se establece en este sentido entre el médico con los enfermos viejos, que prefieren curarse en sus casas con los remedios tradicionales o bien morir en ellas viendo sus campos; con los niños y parturientas que mueren fácilmente ante la resignación de los familiares que acuden al médico demasiado tarde, cuando ya no hay remedio, porque, en muchas ocasiones, han intentado curarlos con remedios inadecuados prescritos por curanderos o por matronas improvisadas e incompetentes del entorno.

Por su parte, los médicos que comparecen en las obras bonavirianas se dividen en dos categorías: los viejos, con muchos años de servicio, habituados a la enfermedad, sin empatía hacia los enfermos y preocupados solamente por conseguir el mejor beneficio económico y los jóvenes que comienzan la práctica de su carrera con ilusión, intentando cambiar la situación, luchando primero contra las administraciones y las autoridades locales que escatiman al máximo los recursos, incluso los medicamentos y las vacunas, y después contra los prejuicios de una sociedad de mentalidad arcaica, hecha a las supersticiones y a los remedios tradicionales, desconfiada y reticente a aceptar cualquier innovación, aunque en ello pueda irle la vida.

La alternativa a tanta miseria y humanidad doliente es para Bonaviri el recuerdo, los montes de Camuti, en Sicilia, la familia, su padre el sastre, el retorno a la infancia o la ensoñación de fabulosos viajes por tierras fantásticas donde solo vive el pensamiento y la enfermedad es únicamente un motivo de debate.

\footnotetext{
Notas

${ }^{1}$ «He ejercido de médico durante seis ańos en Sicilia y durante seis años en el viejo hospital de Frosinone y después en el nuevo. En el viejo, estaba en la enfermería: se trabajaba tres días a la semana de treinta horas de guardia; he aprendido allí a hacer raspados y la asistencia con los fórceps. Esta experiencia me ha hecho descender a las cavernas del dolor humano, y me ha permitido estar en contacto propiamente con el conocimiento profundo del dolor verdadero, y no con el que está en la superficie y que rebota enseguida hacia los problemas políticos, sociológicos, también importantes; el verdadero dolor del hombre es el biológico, de la enfermedad, de la muerte. Mi experiencia ha ensanchado mi visión y mi posibilidad de tener contactos más directos y penetrar mejor en los pliegues más secretos del alma humana. Así pues, en este sentido, existe una relación entre la página escrita y la medicina. Ambas comportan una inmersión en el mundo del dolor humano, filtrado y disperso en todos mis libros». (La traducción de las citas nos pertenece) ${ }^{2}$ «La sangre que le salió era la sangre de todo el mundo».

${ }^{3}$ "Ahora llegó el 9 de agosto y la luna / tuvo una hemorragia retinoica en el ojo derecho,/y allí los lunarios corrieron a la ventana/hacia la tierra, y asustados sobre las dunas/ en el mar Crisium cantaron gallos y grillos/ porque la luna no conseguía atraer la tierra...»
} 
${ }^{4}$ «Este segundo pensamiento se manifiesta y se activa en la fase de duermevela o preonírica. Fuera de ella es inconcebible e inexistente. El origen de fondo de este pensamiento nocturno es, en gran parte, sensorial. Es suficiente autocontrolarse antes de dormirse, para comprenderlo. Basta que durante el pre-sueño, nos sometamos a estímulos cutáneos, o viscerales en un sentido lato (pruritos, somestesias, dolores, simples psicostesias) para que, en un juego de aferencias corticales y de aferencias encéfalo-cutáneas, o viscerales, se presente este pensamiento».

${ }^{5}$ «En este esbozo interpretativo del duermevela me atengo a la hipótesis somático-visceral, excluyendo, entiéndase bien, los dinamismos del sueño. Considero, por ejemplo, el manto cutáneo, verdadera cáscara pericorpórea que nos abraza, se puede, por tanto, considerar un apéndice, expandido hacia afuera, del cerebro; es decir, un verdadero manto mini-encefálico que posee una protofunción arcaica micro-pensante. Por otra parte, no hay que olvidar que la membrana celular tiene una reactividad nerviosa primordial. El cutis podrá poseer también una función primigenia memorizadora».

${ }^{6}$ «De las mil trecientas personas inscritas en la lista de los pobres, el médico municipal, al que sustituía frecuentemente, podía solamente recetar viejos preparados galénicos que se remontaban a hace cien años. - Podéis curar hoy a un niño afectado de broncopulmonía con la infusión de polígala.

—Las infusiones son siempre buenas.

— ¿Y los antibióticos no existen? ¿Si fuese un hermano suyo qué haría? —Doctor, si nos dejamos pillar la mano por la piedad cristiana, se acabó. El ayuntamiento se echará a perder. ¿̨No sabe cuántos millones de déficit tenemos?... Permitid al menos que se pueda prescribir libremente la penicilina». ${ }^{7}$ «El enfermo era hijo de un alfarero que vivía en un callejón estrecho lleno de estiércol y de charcos, pequeños o anchos, en los bordes de las casas. Subí, precedido por el jorobado, por una corroída escalerilla de madera, y allí encontré al padre esperándome teniendo en la mano una lámpara de petróleo. Agarrando esta con ambas manos, enormes por los callos, me acompañó a través de dos habitaciones de paredes desnudas donde estaban colocadas muchas camas... El muchacho estaba sentado en la cama, apoyado en dos cojines altos, rellenos de paja».

${ }^{8}$ «El aire trágico que se respiraba allí dentro es casi imposible de describir. El canónigo N. era delgado, con las mejillas hundidas y los pómulos salientes: los ojos eran lacrimosos y apagados, como los de un animal golpeado por la muerte».

${ }^{9}$ «Sabe, doctor, tengo mis costumbres y mi casa es mi casa. Lo sabe bien usted, no?»

${ }^{10}$ «El doctor con una mirada distante: Ah, sí. Ha ingresado hace pocos días. Tiene una pleuresía húmeda. Enseguida lo vaciaremos. Espero que el pulmón esté indemne. Si es así, curará del todo en un mes. En caso contrario podría tratarse de una tuberculosis que podría durar mucho tiempo. El muchacho estaba muy estropeado cuando ha ingresado».

${ }^{11}$ «- ¿Cómo es que habéis dejado el hospital? —le pregunté después de haberlo visitado. 
-Oh, doctor, ¿ ¿vuecencia sabe lo que significa estar encerrado noche y día en un edificio con grandes pasillos oscuros? Nadie os sonríe, nadie os mira en la cara con compasión, y uno sueña viendo el mucho aire que hay fuera y pensando en nuestros campos».

${ }_{12}$ «En los corredores había cardíacos, bronquíticos, tíficos, o personas afectadas por hemopatías o por hepatitis viral. Normalmente los escolios hemáticos, las expectoraciones, no pudiéndose siempre desaguar por los conductos habituales, eran canalizados hacia las orillas del río vecino. Todo ello, teniendo un régimen torrencial, y teniendo las orillas llenas de ruedas inservibles, de bolsas de plástico, de gatos muertos, de ratas». ${ }^{13} \mathrm{El}$ asilo era una vieja construcción de finales del XIX, dedicada en el pasado a depósito de leńa. En la parte de delante había un jardincillo que terminaba en tres puntas contra las paredes. Aún florecían allí matas de rosas, bajo las cuales se veían lagartijas muertas llenas de pétalos.

${ }^{14} \mathrm{El}$ hospital de los pobres, en Benarés, se encuentra sobre una colina bajo la cual el templo de Nakhan tiene muchas puertas en forma de rosa de los vientos. En las largas columnatas, hechas de piedra, se ve a la diosa Parvati que en poses diversas se une con los toros sagrados... El hospital está constituido por tres pabellones: uno para las mujeres, uno para los hombres, y, finalmente, otro para los viejos que llegan con rostros delgados, y un envoltorio de panecillos de arroz.

${ }^{15}$ «Por cada corredor hay un solo baño, los frascos con las orinas se ponen allí uno sobre el otro por falta de espacio. En algunos cubos se acumulan las placentas de las parturientas de los que chupan sangre las moscas. En una vieja pintura mural se lee: "Si la luna te aluna la cabeza, reza a Shiva. Nuestra alma sufre corrupción eterna”».

${ }^{16}$ «EL MÉDICO (después de un rato responde) Me llamo Michele Rizzo. El edificio es un geriátrico. Por disposiciones recientes además se traen aquí a los niños no nacidos.

DON QUIJOTE (que tiene un escudo azafranado y una mariposa por corbata, color jazmin). Tu lavoro... trabajo me produce una gran tristeza. En este lugar hay solo muerte?... es mortandad infinita. (Agacha la cabeza, clavando la lanza, con la punta rosa, en la tierra).

SANCHO ¡Pobre hijo! ¿No podías haber encontrado otro trabajo? De dónde eres?

EL MÉDICO (para sî) ¿De dónde salen estos? (Después) Soy de Sicilia». ${ }^{17}$ «EL MÉDICO (todavía sorprendido por aquella aparición, después de un meditado silencio dice) Soy un contable de la muerte. Solo consigo recuperar para la vida a algún viejo. Los otros, mientras mueren, mandan reverberaciones sanguinolentas por estas ventanas. Oh, mi gran Don Quijote, al encontrarme me recuerdas mi infancia, hecha de viento, de miseria, de fábulas, de cielos estrellados».

${ }^{18}$ «... la realizada en Mineo, cuando era joven de veinticinco años llegué como médico, tiene una significación particular. Me metía en mi mundo nuevamente pero por otros canales de circulación del dolor y de la experiencia, llegaba, en definitiva, un tiempo enorme en el que fermentaban experiencias milenarias de sabor trágico, de medicina alquímico-mágica, de historia de pacientes ligados uno al otro, por cordones umbilicales... 
Volvía allí como médico, dispuesto a aprehender por coordinadas racionales las secretas resoluciones del dolor campesino».

\section{Referencias bibliográficas}

Bonaviri, G. (2010). L'enorme tempo (a cura di Silvano Nigro). Sellerio. Bonaviri, G. (2009). La ragazza di Casalmonferrato (a cura di Enzo e Sarah Zappulla). La Cantinella.

Bonaviri, G. (2008). L'Infinito lunare (a cura di S. Zappulla Muscarà). Bompiani.

Bonaviri, G. (2007). Silvinia (a cura di Sarah Zappulla Muscarà). Bompiani.

Bonaviri, G. (2004). I cavalli lunari. Scheiwiller.

Bonaviri, G. (1988). Il dormiveglia. Mondadori.

Bonaviri, G. (1986). Ė un rosseggiar di peschi e d'albicocchi. Rizzoli.

Bonaviri G. y Bonaviri G. (1999). E il verde ramo oscillo. Fiabe di folli. Piero Manni.

Nigro S.S. (2010). Un enorme ventenio. Revista Il Resto del Carlino. 14 de diciembre 1976. Texto inserto en el prólogo de Nigro en G. Bonaviri (2010) L'enorme tempo.

Tiberia, R. (2005). Alla ricerca di una poesía biológica. http://www. italialibri.net/interviste/0502-1.html 